\section{EARN £10 FOR EVERY PATIENT}

DPAS has announced the launch of a new initiative that gives practices the means of reaping substantial financial benefits when they switch from a competitor provider to their own DPAS-supported dental plan.

DPAS Solutions 'Voucher Rewards' is a new kind of incentive scheme that offers practices switching from competitor providers to their own DPAS-supported plans a £10 voucher for every patient successfully transferred over a six-month period, building into a fund that can then be used to acquire the external expertise that practices so often need.

This means that a practice with
1,000 patients on plan stands to receive up to $£ 10,000$ to spend on additional services that will further fuel practice growth.

To make the vouchers stretch a little further, participating practices can gain fantastic discounts on support services by using one of DPAS' preferred providers, all of whom have proven track records and intimately understand the needs of dental professionals.

Your entitlement can be used on any resources that you feel will help your practice.

To find out how you can earn a $£ 10$ voucher for every patient you successfully transfer to a DPASsupported plan over a six-month period, call 01747870910.

\title{
SPECIALLY DESIGNED FOR SENSITIVE TEETH
}

GlaxoSmithKline Consumer Healthcare (GSK) is extending its Sensodyne brand with the launch of Sensodyne Complete Protection. With seven speciallydesigned benefits, Sensodyne Complete Protection is targeted to sufferers of tooth sensitivity looking for toothpaste that offers all-round benefits as part of their oral care regime. The new Sensodyne Complete Protection will be available in two variants, Sensodyne Complete Protection and Sensodyne Complete Protection Extra Fresh.

Sensodyne Complete Protection: creates a mineral layer over sensitive areas to shield you from the pain of sensitive teeth; strengthens and rehardens enamel; gives a clean feeling; helps maintain healthy gums; freshens breath; helps control plaque; and helps maintain natural whiteness.

In addition, Sensodyne is launch- ing two new Complete Protection toothbrushes, soft and medium. The brushes are available in four different colour options and feature a small angled head. With soft outer bristles and twisting inner bristles, the brushes are specially-designed for people with sensitive teeth and provide added benefits of plaque removal, enamel and gum cleaning.

Sensodyne Complete Protection products are widely available from June onwards.

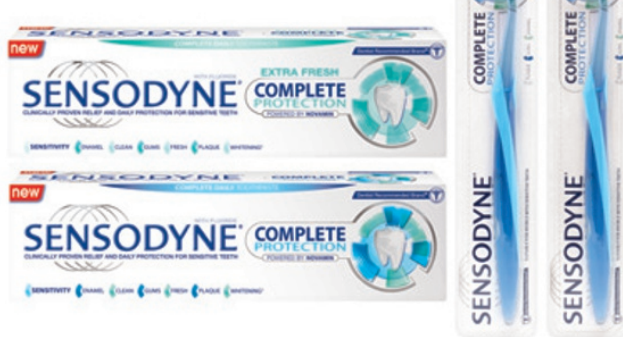

\section{NEW WEBSITE FROM MOUTHWASH RANGE}

Dentyl Active, the scientifically proven two-phase alcohol free range of mouthwash that you can visibly see working, has launched a new website for dental professionals. dentylactivepress.com has been created to provide the latest research and opinion pieces on key oral care matters, brand science and news.

Throughout the year special e-feature news bulletins will be released on the website debating topical oral matters. The first e-feature bulletin focuses on enamel erosion.

Visit www.dentylactivepress.com. 